\title{
ONTOLOGY DRIVEN ANALYSIS OF SPATIO-TEMPORAL PHENOMENA, AIMED AT SPATIAL PLANNING AND ENVIRONMENTAL FORECASTING
}

\author{
Adam Iwaniak $^{\mathrm{a}}$, Jaromar Łukowicz ${ }^{\mathrm{a} *}$, Marek Strzelecki ${ }^{\mathrm{a}}$, Iwona Kaczmarek ${ }^{\mathrm{a}}$, \\ a Uniwersytet Przyrodniczy we Wrocławiu, Wrocław, Poland - (adam.iwaniak iwona.kaczmarek)@up.wroc.pl, \\ (jlukowicz marek.strzelecki)@gmail.com
}

Commission II, WG II/1

KEY WORDS: Spatial, Temporal, Modelling, Planning

\begin{abstract}
:
Spatial planning is a crucial area for balancing civilization development with environmental protection. Spatial planning has a multidisciplinary nature. It must take into account the dynamics of the processes, which could affect the integrity of the environmental system. That is why we need a new approach to modelling phenomena occurring in space. Such approach is offered by ontologies, based on Description Logic (DL) and related to inference systems. Ontology is a system for the knowledge representation, including conceptual scheme and based on this scheme representation of reality. Ontologies can be enriched with additional logical systems. The authors present a way of building domain ontologies for spatial planning, including the representation of spatio-temporal phenomena. Description Logic is supplemented by structures of temporal logic. As a result, the analysis for exploring the topological relations between spatial objects will be extended to include temporal relationships: coincidence, precedence and succession, cause and effect relationship. Spatio-temporal models with temporal logic structures, encoded in ontologies, could be a subject of inference process, performed by semantic reasoners (reasoner engines). Spatio-temporal representations are offered, by so-called upper ontologies, such as GFO, BFO, OCHRE and others. Temporal structures provided in such ontologies, are useful for the analysis of data obtained from environmental and development monitoring systems and for description and representation of historical phenomena. They allow creating the models and scenarios of expected spatial transformation. They will support analysis for spatial development design, decision-making in spatial planning and forecasting of environmental impact.
\end{abstract}

\section{INTRODUCTION}

The international and local communities have become increasingly aware of the need for responsible managing of the environment resources. People recognize the problems and risks associated with uncontrolled spatial development. Society expects the spatial planner or environmental expert to prepare design solutions that take into account a credible analysis of the impact of spatial development environment. So far, planners, environmental professionals formulated their forecasts about the changes of the environment based on expertise in their field, their own experience and intuition. They receive today a set of design and forecasting tools for creating scenarios using objectively defined methodologies.

\section{BACKGROUND AND CONTEXT OF PROBLEM}

\subsection{Spatial management tasks}

The purpose of planning is to solve the conflict between civilization development and environmental protection. It requires continuous diagnosing of environment immunity against settlement and economic pressures. Planning is interdisciplinary and continuous in time. It must take into account the dynamics of the processes affecting the integrity of the natural system.

\subsection{Limitations of existing analysis methodologies}

Traditional methodologies based on the GIS are robust and have a high performance. They have, however, many limitations. They often do not provide answers to more complex questions. Forecasting changes in the environment requires the processing of heterogeneous data with different schemas that integration causes many problems. This is the reason that forecasting of the environment changes, are made in the traditional, not an automated way.

\subsection{Semantic Web and new ideas of reality description}

This implies a need for more flexible methods of analysis and design, providing reality-modelling tools. Such hope gives Semantic Web technologies. Systems of formal description logics, temporal logic and probability, add to the Semantic Web, new opportunities, suitable to describe the dynamic phenomena occurring in the spatial planning and the environment. Such a system would be useful to support planning decisions in the design process and predict their impact on the environment and the future state of spatial development.

Semantic Web uses ontologies for describing the real world. Ontologies spread before the idea of the Semantic Web, but in this environment, they prove their efficient application. Ontology (from Greek, wording to ov means "that which is", which we can interpret as being; in genitive, ovtoc could be used as "[that what concerns] the being"). Gruber describes it in short way: An ontology is a specification of $a$ conceptualization. (Gruber, 1995) Ontology is based on a particular view at the world: ontology asks what an entity is, what the essence of it is, and which mode of existence it has. (Herre, 2010).

\subsection{Available ontologies for spatio-temporal modelling}

The interdisciplinary character of spatial planning problems results in the need for the modelling for a large number of classes and objects and a large number of their mutual relations. The models created for particular branches constitute the socalled domain ontologies (domain-specific ontologies). The 
following members of this group need to be mentioned: Basic Geo spatial vocabulary (WGS84 lat/long) - simple ontology of spatial objects ${ }^{1}$, W3C Geospatial Ontologies - OGC standard ontologies $^{2}$, GeoRSS - RSS extension for geospatial problems ${ }^{3}$, NeoGeo Vocabulary - a dictionary of geospatial and topological problems with relation to OGC standards (GeoVocab.org) ${ }^{4}$ etc. Upper ontologies, called also fundamental ontologies, top level ontologies or upper ontologies are another important tool. They are used as dictionaries including the taxonomies of the classes common to numerous branches. They allow for an applicationindependent description of reality. The most important solutions of this sort include: Dublin Core ${ }^{5}$, SUMO ${ }^{6}$, SWEET ${ }^{7}$ and many others. Top-level ontologies include formal ontologies. Among upper ontologies important role play formal ontologies. Ontologies based on the aforementioned formal systems include DOLCE $^{8}$, BFO (Basic Formal Ontology) ${ }^{9}$, GFO (General Formal Ontology $)^{10}$ and others. It is worth mentioning solutions suitable for analyse of spatio-temporal issues present in ontological representation of reality. They are used in the extraction of facts relating to space from semantic data. As example, we could point solutions for Qualitative SpatioTemporal Reasoning. (Renz et al. 1999) (Gerevini et al. 2002)

\section{SEMANTIC DESCRIPTION OF CHANGING WORLD}

\subsection{Ontologies as models}

Model and metamodel of reality. The reality, even if we consider it from the perspective of a chosen domain, it is always a complex system. Therefore, we are forced to reduce the set of entities and describe their characteristics. Each description is therefore a model, an abstraction that separates our perception of the real world. This problem is solved in different ways, depending on the philosophical assumptions. To simplify this problem, we have to choose between three approaches: realistic like BFO (Grenon et al. 2004), the approach considering world at different levels of reality, like GFO (Herre, 2010), and perceptual and cognitive bias like DOLCE (Masolo et al. 2003). Building an abstraction of reality there is the issue of model and meta-model (called in DOLCE as meta-language). Division into categories (class types), properties (predicates), and concrete individuals is solved in various ways, as a result of different philosophical assumptions. Usually, all the elements of the model and meta-model, which are used to describe the facts, are termed entities. In some systems, a distinction is made between universals and Particulars. In some systems, a distinction is made between universals and particulars. Usually, the universals include classes and properties (e.g., relationships), and particulars include instances of these classes, objects that reflect the real beings. The "model - meta-model" relationship, corresponding to "particular - universal" relationship can be multi-level. This is similar to the concept of "punning" of OWL2.

1 Basic Geo (WGS84 lat/long) Vocabulary; http://www.w3.org/2003/01/geo/wgs84_pos\#

2 W3C Geospatial Incubator Group (GeoXG)

http://www.w3.org/2005/Incubator/geo/XGR-geo-ont/

3 GeoRSS; http://www.georss.org/Main_Page

4 NeoGeo. http://geovocab.org/

5 DCMI Home: Dublin Core Metadata Initiative (DCMI) http://dublincore.org/

6 Suggested Upper Merged Ontology (SUMO) http://www.ontologyportal.org/

7 SWEET - Semantic Web for Earth and Environmental Terminology; http://sweet.jpl.nasa.gov/ontology/

8 DOLCE - Descriptive Ontology for Linguistic and Cognitive Engineering; http://www.loa.istc.cnr.it/

9 BFO The Basic Formal Ontology (BFO); http://www.ifomis.org/bfo

10 GFO General Formal Ontology (GFO) http://www.onto-med.de/ontologies/gfo/

\subsection{Multiview description of reality}

Extract the model from reality, and the subsequent reduction of qualities describing reality, needs to reconcile the contradiction between the desire for the simplest model useful for a particular purpose, and the need for model embracing interdisciplinary issues. For these problems refers precisely BFO. Methodology of BFO is realist, perspectivalist, fallibilist, and adequatist. Realism means accepting that reality exists independently of us. Beings and elements of reality are independent of our representation of this reality. On the other hand, perspectivalism means that we can create alternative, equally legitimate perspectives of description of reality. Limiting the legitimation of these perspectives is a result of other assumptions (fallibilism and adequatism) (Grenon et al. 2004).

\subsection{Adding time to description of space}

Description of the dynamics of the change of space can be introduced in several ways.

Spatio-temporal representation in SDI. In traditional data collection technologies have been used two approaches. For data representing objects or phenomena evolving over time in a continuous manner (eg, population, forest cover, GDP, etc.) were used data series. They permit analyzes using well-known statistical tools. The second approach, which refers to a persistent objects (e.g. land, buildings), was the addition of the history of the object. It could be done by attributes registering the moment of object creation and (potentially) the moment of object destruction (Quak et al. 2011). Registration of object versions can be considered as a more advanced method ( $\mathrm{Li}$ et al. 2008). History of objects sometimes is enriched by the introduction of predecessors and optionally successors of object. Applying the evolution of these objects require a separate analytical systems, not built into the model (Berman, 2009) (Guangfa et al. 2005).

Spatio-temporal RDF graph. A similar approach, but using semantic-based structures, includes a temporal RDF graphs. Properties forming the graph relationships are enhanced with additional properties that define the time snapshot (timeSlice) or time interval (timeInterval), in which the graph is up to date. The dynamics of the phenomena is represented by a series of graphs that describe the successive moments or intervals when phenomena exist (approach similar to time series, but representing whole graph structure).(Gutierrez et al. 2007)

Temporal approach in upper ontologies (formal ontologies). Systems based on ontologies represent quite a different approach. Ontologies describe objects and their classification, taking into account their constituent characteristics. That is, the ontology defines the object due to its specific features. Bearing that in mind it is possible to build a comprehensive system of logic that classifies objects by their behaviour over time. Currently, we estimate that among all the solutions, the greatest benefit for modelling spatial issues can give BFO. We do not exclude other potential solutions, but at this point the BFO approach seems to be the most promising. (Bittner et al. 2009)

Spatial and temporal operators. The logical semantic representation is built on relationships. Set of relationships that describe the relative positions of objects and their chronological dependence is needed for the phenomena described both in space and in time. Ontologies provide a formal structure describing their own time and space. In such frame we could involve mereological relationships, mereotopological and temporal contained in the various logical systems (Hahmann, 2013). Spatial and temporal analyses can be carried out using specially developed for this purpose distinct "calculi": RCC - 
Region Connection Calculus for topological relationships (Renz et al. 1999), Allen's operators for temporal relationships (Allen, 1983) (Gerevini et al. 2002), and others.

\subsection{Representation of dynamic reality in formal ontologies}

The distinction between objects according to their behavior in time. Almost all formal ontologies distinguish between objects, which are persistent over time, called continuants or "happening" at the time (running, flowing), called the occurents. They are also called, respectively endurants and perdurants. (Figure 1)

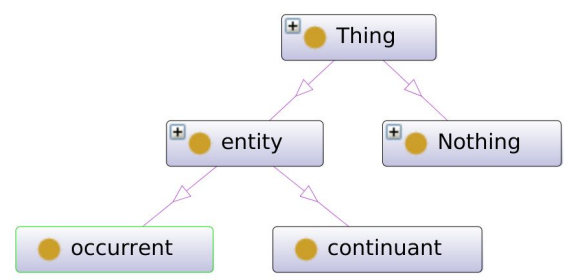

Figure 1. Top-level distinction between continuants and occurents

Endurants include physical objects, for example: building, road, parking, tree, lake, river. (Figure 2) But they can also be abstract objects that do not physically exist. The parcel (plot) can't be seen in the physical space, but it affects its shape (fences, location of the building). It really is an object existing in the land cadastre database. However, despite this plot is an endurant. Perdurants are events or processes, but some systems, like BFO, extends this list by temporal and spatiotemporal regions. (Figure 3)

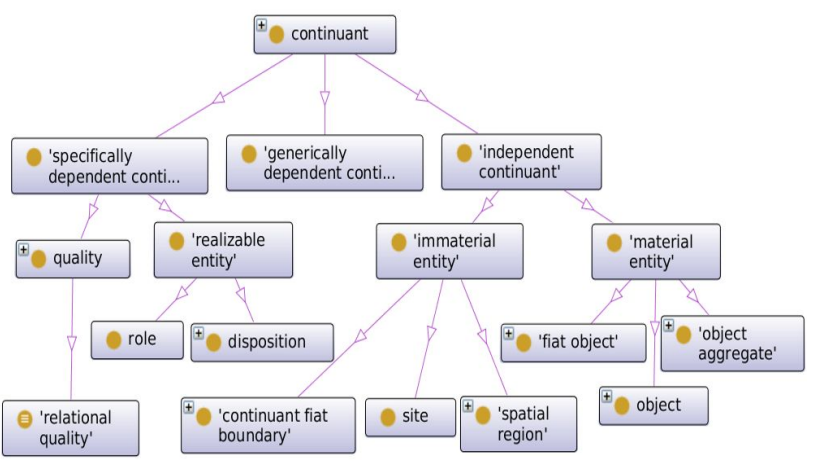

Figure 2. Hierarchy of continuants on the example of BFO

Endurants include physical objects (building, road, parking, tree, lake and river). However, they can also be abstract objects that do not physically exist. The parcel (plot) cannot be seen in the physical space, but it affects its shape (fences, location of the building). It really is an object existing in the land cadastre database. However, despite this plot is an endurant. Perdurants are events or processes.

Each of the formal systems provides another approach to the distinction between endurants and perdurants. According to DOLCE endurants are wholly present (i.e., all their proper parts are present) at any time they are present (Masolo et al. 2003). In this definition, the emphasis is put on mereological aspect of persistence. Endurant is characterized by the fact that it is not necessary to view it in the whole time, as even a snapshot be a representative for this entity. In contrast to this, a description of the process involves seeing it in the whole time interval in which it extends. Snapshot will show only a part of process and not be representative for this entity.

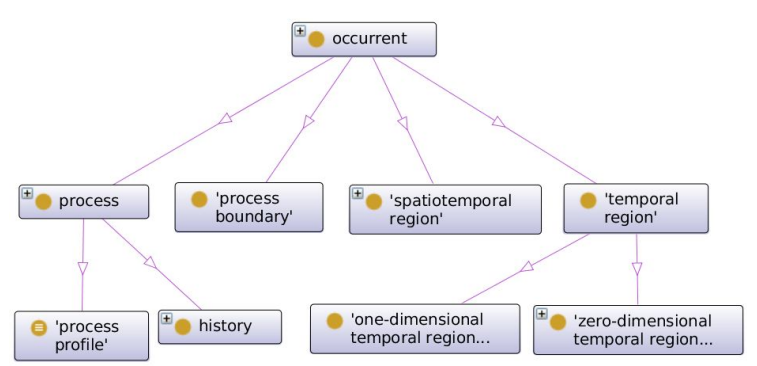

Figure 3. Hierarchy of occurents on the example of BFO

To simplify this distinction, continuants / endurants "persist" at the time, and occurents / perdurants "happen" at the time. (Masolo et al. 2003). According to BFO occurents change state of continuants, while the occurent can "happen" only in connection with some continuant (Grenon et al. 2004). This approach affects the core of this distinction. From a logical point of view, "persistence" of continuants during the time has a passive character. However, the "happening" with influence on continuants state, makes occurent "active". Change of continuant state is caused by some occurent. On the other hand, occurent cannot exist without some continuant - a continuant is the bearer of occurent. (Grenon et al. 2004).

Relations among endurants and perdurants. The former are strongly associated with the three-dimensional space, while the other are described temporarily or spatio-temporally. This makes it difficult, to study their mutual topological and temporal relationships, because these objects are like from different worlds.

There are different ways to solve that problem in the various ontologies. The formal system of BFO creates separate ontologies for continuants and occurents: respectively SNAP and SPAN ontologies. SNAP ontology models only continuants as SNAP entities. SNAP ontology is a snapshot of the state of reality in three-dimensional perspective. SPAN and the models occurents, as SPAN entitities, presenting their temporal and spatial boundaries (four-dimensional perspective). BFO, however, requires additional ontologies, which reconcile these areas in a coherent framework - these are trans-ontologies.

Persistence of objects and their change. In order to study stability and change objects in time, a formal system of BFO, which divided the objects SNAP and SPAN, provides some trans-ontologies (SNAP-SNAP, SPAN-SPAN i SNAP-SPAN). They are used for reasoning about the dynamics of phenomena involving together endurants and perdurants.

Ontology SNAP-SNAP allows the reasoning involving comparisons about states at different times (snapshots, temporal-slices). There is here a certain analogy to temporal RDF graphs. The main task of ontology SNAP-SNAP is to discover the changes. They refer to the state of endurants and can be divided into 3 groups (Grenon et al. 2004):
1. Qualitative Change
2. Substantial Change
3. Spatial and Locational Change

Qualitative Changes include transformations that preserve the continuity of the identity of objects, but describe the quality changes (changes in characteristics) profoundly affect the nature of the entity. Group of Qualitative Changes includes Change in determinables, Qualitative creation and Qualitative destruction. 
Substantial Change is a change that results in termination of the object. A change of this type describes an example glass sphere, which crashes down on the floor. BFO accurately describe variants of these changes: budding, absorption, separation, unification and distinguishes between objects according to their substrates and products of these changes (dissective and cumulative).

Spatial and Locational Change, does not necessarily mean in the physical sense of movement. They include the changes to the boundaries of a given country (Grenon et al. 2004).

The final structure of the ontology SNAP-SNAP is genidentity. Since SNAP ontologies look like snapshots (time slices), it is important to show the dependencies and consequences of mutual preceding and succession of objects. Genidentity provides information about the trans-ontological filiations of objects (Grenon et al. 2004). Its role is similar to that used in traditional GIS determination of object predecessors and successors.

Mereology and mereotopology. SNAP ontology provides the structure to describe the spatial aspects of the position of objects, their size and shape, and the relationships between them, including part-whole relationships as well as the relative position of spatial relationships. In this case, the behaviour of entities depends on whether they are dissective or cumulative.

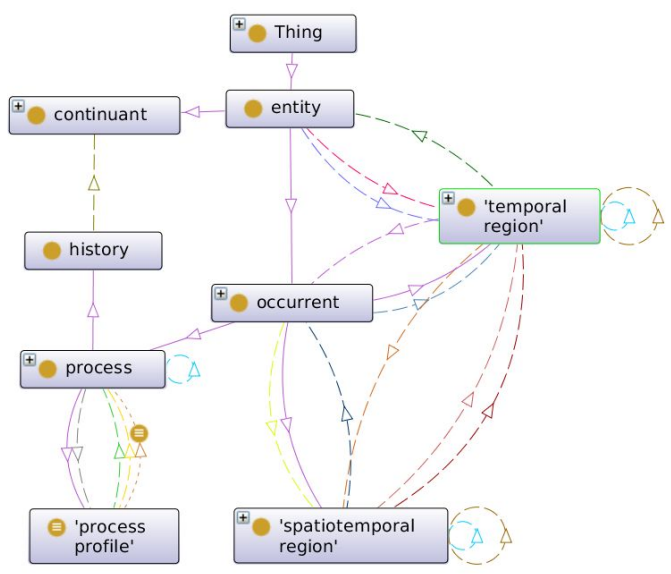

Figure 4. BFO SPAN structure

Reasoning about the dynamics of the phenomena under SPAN ontology is possible within a single ontology, covering a specific time interval. It operates on perdurants represented as SPAN entities. The succession of time is also included in this ontology. The most typical entity in SPAN is a process. Characteristics of the process is described by the 'process profile', which is subclass of the process. 'Process profile' could describe rate of change of entity's state, caused by process. 'History of life' is subclass of the process class too. It describes directly the changes of the continuant. (Figure 4)

Entities such as the temporal region and the spatiotemporal region are also included in the facilities SPAN.(Grenon et al. 2004) These two basic types allow you to create a whole range of complex descriptions of space and their parts (Spatiotemporal parts) (Grenon, 2003). Regions and parts can be analysed by examining their mereological and mereotopological relationships. However, when you have to go outside this range, you need trans-ontology SPAN-SPAN.

Trans-ontology SNAP-SPAN combines issues of persistent objects and processes. Ontology SPAN-SNAP does the same in the opposite direction. Relations endurant - perdurant, spatial region - temporal region, spatial region - spatiotemporal region, are the main issues described by the ontology. The other issues are mereological and mereotopological relationships in fourdimensional space. This ontology creates a unique relationship between the SNAP entity and a specific SPAN entity, which is the 'history of life' of this object including its beginning and end (Figure 5). This allows you to create a relationship showing the objects SNAP in particular temporal region, as existence during a period of time. Mutual relations between continuants (represented here as SNAP) and occurents (SPAN entities) include the role of continuants as bearers of occurents ane vice versa impact of occurents on the state of continuants. The entire family of trans-ontological SNAP-SPAN relationships includes: participation, initiation, termination, creation, destruction, sustenance, deterioration, facilitation, hindrance and others (Grenon et al. 2004).

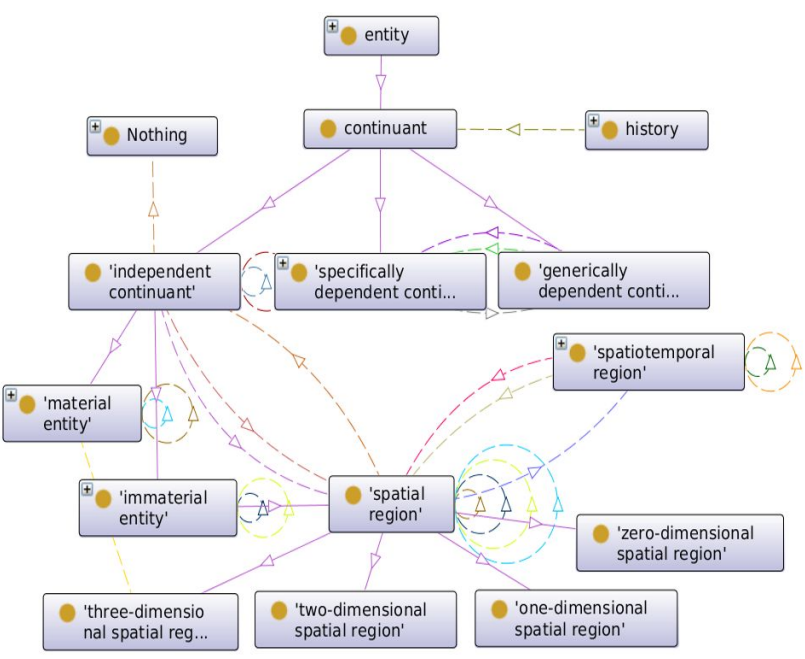

Figure 5. BFO SNAP-SPAN relationship

\subsection{Possibilities of application to spatial management issues}

The use of the model provided by the BFO, was made by the choice of the interesting objects from the domain of spatial management and entering them into time-spatial meta-model. Example took a set of selected classes related to spatial planning and environmental protection. In the first stage, we intend to show how the different classes of objects are mapped to the structure of the BFO and how to model its dynamic behaviour. The second phase aims to consider how to determine the expected development of the space state. The following classes were chosen for analysis:

objects of land cadastre,

- $\quad$ objects represented land cover - they will allow the description of the actual state of the environment and the anthropomorphic impact,

- $\quad$ real objects representing some fragments of the land (with meaning of the earth surface).

The choice of a class of objects in this example has been intentionally limited to have the opportunity to present their complete structure relationship. In this set, such classes are chosen, which actually remained in the mutual logical relationships.

Real objects as continuants. All of the above objects are entities of continuants (endurants). In addition, they are entities 'independent continuant' that reflect the real phenomena. The real does not necessarily mean the physical. Although ontology BFO, unlike DOLCE, does not make this distinction, it must be conscious that the real object may be either abstract. 
The BFO distinguishes between categories of 'material entity' and 'immaterial entity'. Contrary to intuition, not fully reflect the distinction between the physical object and the abstract. For example, the fiat objects' are classified as 'material entity'. They reflect various planned, legal, conceptual division of physical objects (division of the globe into hemispheres, the division of the brain into regions). For this type of objects you can include all the created spatial divisions that are presented on different kinds of maps: surveying, soil, hydrography. Therefore, parcels, land use units, building footprints, and even units of land cover were included in this category. Category 'object aggregate' is also abstract, although it is a sub-category of 'material object'. It is a structure analogous to the aggregation in the object oriented modelling or collection in RDF.

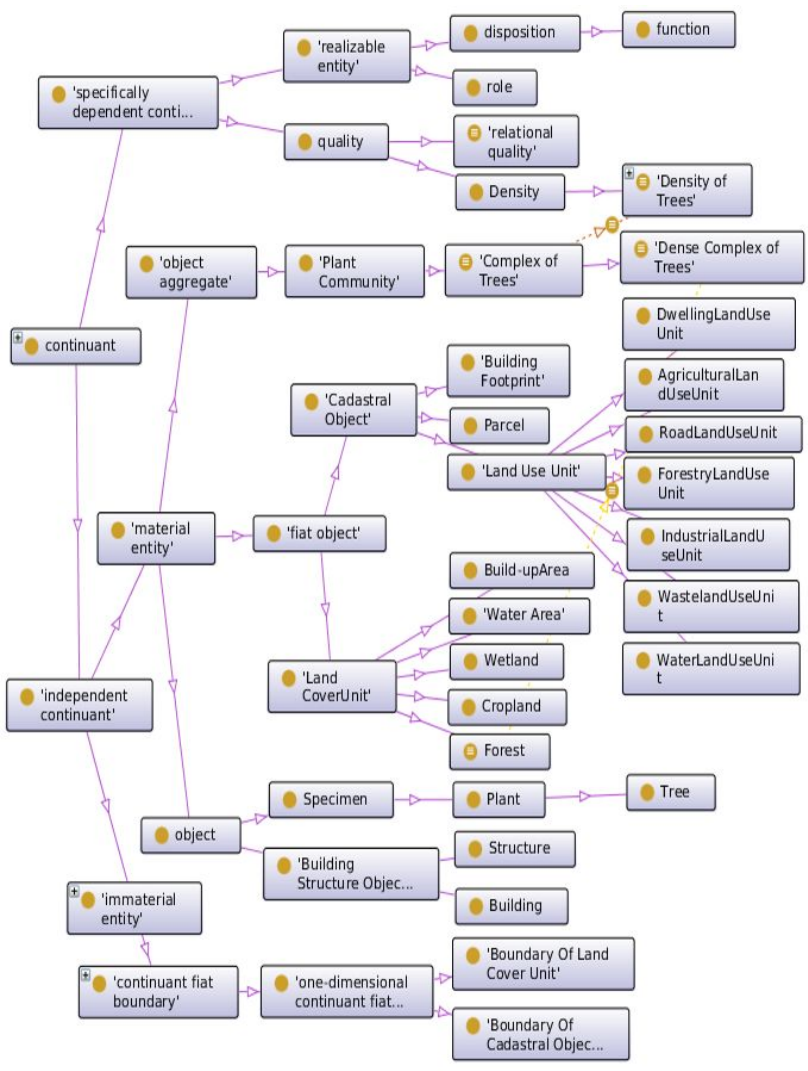

Figure 6. Extending BFO continuant classes by real world object classes representing spatial management issues

Modelling using the BFO can take two strategies. The first would be implemented through direct instantiation of universals of BFO, making an implicit classification by assigning the appropriate qualities. The second is the explicit extension of the model by user subclasses. In this example, we chose the second option. Since we use the version of BFO ontology provided in OWL2 DL, in Description Logic formalism, a natural solution seems to be to use expandable class hierarchy using the relation rdfs: subClassOf. The above-described classes of spatial objects are associated with the hierarchy of formal category of endurants in BFO. (Figure 6)

Classification based on properties. These objects are described by properties. They determine the characteristics of objects and their state. The analysis focused on subjects related to widely understood "forest". From the environmental point of view, it is a floral complex, forming the environment for a number of characteristic floral and faunal communities, creating together forest biocoenosis. When considering land cover, it is area with high density of trees. Natural aspect is essential for the sustainability and the state of this complex. The impact on the natural equilibrium of this ecosystem will gradually fading of the forest. Crucial for the classification of this object, it will decrease density of trees. Upon reaching a certain value, you can not now speak of the forest, as a de facto form of land cover.

Thus, objects of class 'land cover' will be held for the entire period of his life as a 'member part' object of class of 'Complex of Trees', which is a subclass of 'object aggregate'. However, not every object of the class of 'Complex of Trees' will determine that there is an area of forest. This will be such 'Complex of Trees', which will be held for the entire period of his life, quality 'high density of trees'. Restriction (Expressed in Manchester Syntax) "'has quality at all times' value 'high density of trees"' assigns a entity to the class of 'Dense Complex of Trees' subclass of 'Complex of Trees'. Forest is a class of objects that have a classification criterion: "'member part has at all times' only 'Dense Complex of Trees."' If, in due to harmful influence of surroundings will decrease density of trees, which is no longer be of 'high density of trees', the forest will cease to exist, and the area related to it, will cease to be an area of forest.

Category 'Density of Trees', which is the "determinable universal" has three instances (tropes): 'high density of trees', 'medium density of trees' and 'low density of trees'. As you can see, these are the categories of a qualitative not quantitative type. They might be linked to another feature of a quantitative type that will prejudge which trope (determinate), will instantiate this determinable. In this way, you can come to individual objects, 'trees' that will create collections used to evaluate the tree density. Going down to such level of detail, we use a feature of BFO, which is the granularity. This corresponds to the well-known GIS change of scale, involving a change in resolution imaging phenomena. With decreasing scale on map series, is made generalization of map content, corresponding to the construction of 'aggregate objects', which occurs in BFO.

This issue requires more research, but we believe that the quality-quantity relationship should be based on fuzzy intervals of values instead of crisp intervals. Keeping of qualitative features enable the use of qualitative reasoning, which allows process that is more efficient.

Modelling object changes - role of processes and events. When you have identified the objects and their attributes, define what is responsible for the dynamics of these processes. Continuants are beares of occurents. That is the state of spatial objects of the real world, is subject to change as a result of processes. The whole appeal of Spatio-temporal ontology is to capture the process, not only in statistical form, but above all in the cause-and-effect relationship. The nature of the process such as the rate of change is described by occurent 'Process profile'. As example, it could be the rate of deforestation. This allows you to specify mode and strength of the impact of the occurent on the state of the continuant. In the tested model are defined following categories of occurents, as shown on Figure 7.

Having defined processes and process profiles, we can reconstruct the history of changes in objects. When the changes of tropes reach states defined by the criteria of classification, this may occur qualitative change or substantial change. This could have happened in the past, but it can also occur in the future. With SPAN ontology that describes the processes at time intervals, we know history of live of given objects. History of life can be extrapolated into the future, to infer future states of objects. This will provide knowledge about possible future facts, that will result from the changes and reclassification of objects. Ontological description allow the use for this purpose available reasoning engines. 


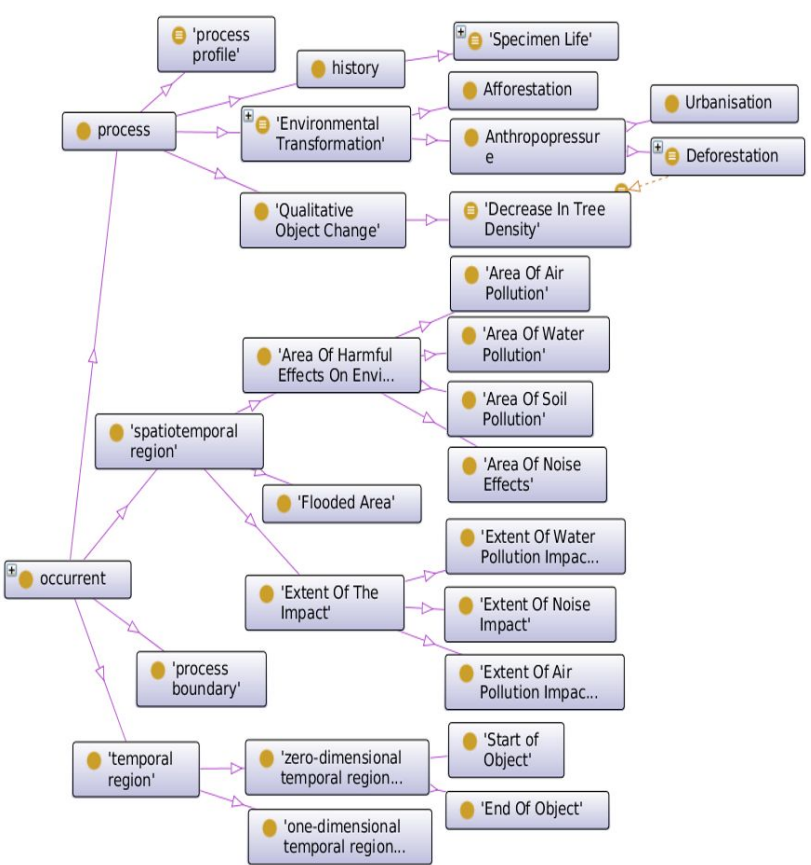

Figure 7. Extending BFO occurent classes by classes representing processes and events in spatial management issues

\section{CONCLUSIONS}

The main problem in spatial management is to create structures that will effectively represent the dynamic behaviour of spatial objects. Traditional GIS systems are characterized by high technological maturity, with a very efficient storage systems, extraction and processing. Their problem is an artificial model of reality to translate information and computing systems. A new approach for modelling of spatial phenomena using the Semantic Web technologies and formal ontologies offers new flexible modelling tools. Ontological approach, using Description Logic with meta-language provided by fundamental ontologies allows the modelling of spatial phenomena, such that they are, or at least like we can see them and understand. We can analyse the characteristics of the real phenomena and objects, giving them their own character, without worrying about technological limitations and application schema. Maintaining an appropriate mathematical formalism and logical, there will be a way to transform the model into a relational or object-oriented models.

\section{FUTURE WORK}

Analysis of the potential use of formal ontologies in the spatial management domain suggests possible directions for further research. First of all, we intend to test the application of other top-level ontologies. Within range of the BFO, we are going to explore the possibilities of representation of spatial data at different scales, using the concept of granularity. Another issue, is to generate data models defined in formal ontologies schema from data stored in traditional database systems, and shared in the traditional SDI (relational and object-oriented). Next group of issues is greater use of temporal logic formalism (Linear Temporal Logic LTL or Computation Tree Logic CTL) and modal logic. The most promising opportunities gives an introduction to spatial models of probability factor. While introducing the extrapolation process, there is an expectation to consider future scenarios in a probabilistic manner. There are available OWL language extensions, such as Bayes OWL ${ }^{11}$ and PR-OWL ${ }^{12}$, providing constructs based on Bayesian Network.

\section{REFERENCES}

Allen. J.F., 1983. Maintaining knowledge about temporal intervals. Commun. ACM, 26(11) pp.832-843,

Berman, M.L., 2009. Modeling and Visualizing Historical GIS Data. Harvard University, http://www.fas.harvard.edu/ chgis/work/docs/papers/CGA_Wkshp2009_Lex_9apr09.pdf

Bittner, T., Donnelly, M., Smith, B., 2009. A spatio-temporal ontology for geographic information integration. International Journal of Geogr.Inf.Science, Vol. 23, pp. 765-798

Gerevini, A., Nebel, B., 2002. Qualitative Spatio-Temporal Reasoning with RCC-8 and Allen's Interval Calculus: Computational Complexity. Proc. 15th ECAI02, pp. 312-316

Grenon, P, 2003. BFO in a Nutshell: A Bi-categorial Axiomatization of $\mathrm{BFO}$ and Comparison with DOLCE, IFOMIS REPORTS

Grenon, P, Smith, B. 2004. SNAP and SPAN: Towards Dynamic Spatial Ontology. Spat.Cog.Comp., Vol. 4, pp. 69-104

Gruber, T., 1995. What is an Ontology? International Journal of Human-Computer Studies, Vol. 43, Issues 4-5, pp. 907-928

Guangfa, L.I.N., Wanli Huang, Huixian Jiang, Youfei C.H.E.N., 2005. A Methodology of modelling object history oriented to spatio-temporal reasoning. Proceeds.of the Intern.Symp.on Spatio-temporal Modeling, etc..., Vol. 2005

Gutierrez, C., Hurtado, C.A., Vaisman, A., 2007. Introducing Time into RDF. IEEE Transactions on Knowledge and Data Engineering, Vol. 19, No. 2, p. 207

Hahmann T., 2013. A Reconciliation of Logical Representations of Space: from Multidimensional Mereotopology to Geometry. www.cs.toronto.edu/ torsten/ publications/Hahmann_PhD_thesis.pdf

Herre, H., 2010. General Formal Ontology (GFO) A Foundational Ontology for Conceptual Modelling. Media, Vol. 2, Issue: 15 , pp. 1-50

Li, Y.D., Tong, X.H.,Liu, M L., 2008. A Unified Version-Based Spatio-Temporal Data Model. Archives, pp. 103-108

Masolo, C., Borgo, S., Gangemi, A., Guarino, N., Oltramari, A., Schneider, L., 2003. The WonderWeb Library of Foundational Ontologies. WonderWeb Deliverable D17, Preliminary Report.

Quak, W., de Vries M., 2011. Topological and temporal modeling in GML. http://www.gdmc.nl/publications/2005/ Topological_Temporal_Modelling_GML.pdf

Renz, J., Nebel. B., 1999. On the complexity of qualitative spatial reasoning: A maximal tractable fragment of the Region Connection Calculus. Artificial Intellig., Vol. 108, pp. 69-123.

This research work has been supported through the projects granted by the National Science Centre, Poland - projects numbers: UMO2012/05/N/HS4/00642 and UMO-2012/05/B/HS4/04197

11 Bayes OWL Reference;

http://www.csee.umbc.edu/ ypeng/BayesOWL/manual/index.html

12 PR-OWL: A Bayesian extension to the OWL Ontology Language; http://www.pr-owl.org/ 